Int. J. Dev. Biol. 50: 359-365 (2006)

doi: $10.1387 / \mathrm{ijdb} .052039 \mathrm{kv}$

\title{
Tissue morphogenesis: a surface buckling mechanism
}

\author{
KONSTANTIN Y. VOLOKH* \\ Departments of Orthopaedic Surgery and Mechanical Engineering, Johns Hopkins University, Baltimore, USA
}

\begin{abstract}
Surface patterns can emerge during growth of anisotropic tissues because of surface buckling. This morphogenetic scenario is examined in the present paper based on a simple phenomenological theory of tissue growth. In particular, we show that constrained growth can lead to tissue compression, which in turn may result in surface buckling of the tissue. The latter means the appearance of wavy patterns on the surface. These patterns decay away from the surface. It is interesting that the critical magnitude of the parameter of mass supply, which corresponds to surface buckling, is independent of the pattern wavelength and various patterns can generally be generated in growth. Results of theoretical analysis show that the surface buckling scenario is realistic if the growing tissue matches the following two conditions. First, compression should appear during tissue growth. Second, the tissue should exhibit strong anisotropy. The former condition does not necessarily mean geometric constraints: inhomogeneous growth or material inhomogeneity and anisotropy can lead to the appearance of compressive stresses. The latter condition is typical of some tissues with fiber reinforcement in planes parallel to the surface. In the latter case, the tissue material is much softer in the out-of-plane direction than in plane. The creation of patterns by restraining tissue growth and forcing the surface to buckle represents a challenging experimental problem.
\end{abstract}

KEY WORDS: morphogenesis, patterning, buckling, growth, tissue

\begin{abstract}
$" /$...assert that the process of tissue formation is in many ways the cellular equivalent of molecular self-assembly and that the appropriate language in which to analyze morphogenesis is that of the differential equation...”
\end{abstract}

$$
\text { Jonathan Bard, «Morphogenesis» (1990) }
$$

\section{Introduction}

Bard (1990) discusses three viewpoints on tissue development. They are the genetic view, the epigenetic view, and the middle view. According to the genetic view, the tissue formation is entirely determined by the DNA-coded information stored in the genome and interpreted by cells. According to the epigenetic view, the development of the tissue structure is affected by its physical environment. The middle view is a mixture of the first two views, which emphasizes that the weight of a specific scenario, genetic or epigenetic, depends on the case under consideration. It should not be missed that the literature provides theoretical and experimental arguments in favor of any scenario that are equally convincing or vague (depending on a reader background).

Strengthening arguments in favor of the epigenetic/middle view Newman and Muller (2000), and Cowin (2004) suggest three epigenetic (sub-) views on the tissue development. The first one is the interactions of cell metabolism with the physicochemical environment within and external to the organism. This view is rooted in the celebrated work by Turing (1952) where the idea that the diffusing patterns of reacting chemicals can form steady-state heterogeneous spatial patterns was put forward. Many researchers have further developed this idea and the summary of results can be found in Murray's book (2003) where chemo-mechanical coupling is also considered. The second view associates the epigenetic mechanism with interactions of tissue masses with the tissue's physical environment. In the case of bone, for instance, it is thought that mechanical loads are sensed by osteocytes, which transform the mechanical signal in a biochemical one mechanotransduction. The latter, in its turn, changes the bone development program. Such scenario is (arguably) known by the name of Wolff's law (Cowin, 2001). The third view associates the epigenetic mechanism with interactions among the tissues themselves. During such interactions, compressive stresses can appear which lead to the mechanical buckling of the tissue structure accompanied by the formation of the buckling modes - morphogenetic patterns. Green (1999) and Steele (2000) and their associates who correlated tissue morphogenesis with the problems of buckling of beams, plates, and shells on elastic foundation ad-

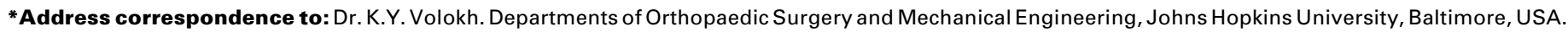

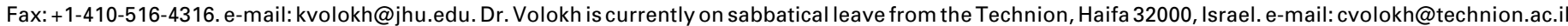


vanced this scenario.

We consider a theoretical argument, which can potentially unify the different views on epigenetic morphogenesis. We formulate a continuum mechanics theory of tissue growth which is different from many existing theories in that it is simple, microstructurally motivated, and free of hidden variables, i.e. experimentally tractable (Volokh, 2004a,b; Volokh and Lev, 2005). Then we apply this theory to a model problem of tissue growth allowing for an analytical solution. We show that restrained growth may lead to the compressive stress in the bulk tissue, which can initiate the phenomenon known as surface buckling when only a material layer close to the surface buckles producing morphogenetic patterns while the whole bulk tissue remains undisturbed. Our approach has somewhat common with all three views on epigenetic morphogenesis. First, its mathematical structure-partial differential equations - is similar to that of Turing-Oster-Murray including balance equation of the reaction diffusion type. Second, the terms corresponding to tissue remodeling, connected to mechanotransduction, are included in the formulation of equations (though this issue will not be explored in the present work yet) similar to the adaptive growth theories (Cowin and Hegedus, 1976). Third, the possibility of the growth bifurcation in the form of elastic buckling is considered resembling the ideas of Green and Steele on structural instability as a source of patterning. It is worth noting that all these features are the natural outcome of the proposed theory without additional ad hoc assumptions.

\section{Governing equations of mechanics of tissue growth}

Biological or biochemical mechanisms of growth are not well understood though a plenty of scenarios exist in the biological literature. There is no doubt that biochemistry is the driving force of tissue growth. Understanding the biochemistry of growth is most desirable. Biochemistry can explain whya tissue grows. That is not enough, however. It is also necessary to know howa tissue grows. The latter means macroscopic description in terms of the macroscopically measurable parameters. The macroscopic parameters of tissue growth seem to be self-evident: mass and form (configuration). Observations on plants and animals, including human beings, during the lifetime give the strict experimental proof of the change of mass and configuration.

The assumption that the continuous deformation and mass flow can describe growth of living bodies is central for further development. To make sound this assumption, geometry of growth should be analyzed qualitatively. Sharp distinction between the real physical material and the mathematical concept of materialpointshould be kept in mind. This distinction is illustrated in Fig. 1 where the conventional deformation (on the left side) is compared to the deformation-growth (on the right side). It is assumed that material volume increases during the deformation while the 'number' of material points remains the same. The concept of the material point is purely mathematical. Material points do not exist: they are mathematical abstractions. Material always occupies some volume. One means a very small material volume saying 'material point'. Every material point enlarges during the deformation together with the whole body as it is shown in the figure. Under 'higher resolution' it can be seen that a material point is a very small physical volume, which in the case of living tissues includes cells, molecules, and various tissue particles. Conventional deformation preserves the same material particles inside the considered referential point-volume, the referential mass density is not changing during deformation: $\rho=$ constant. This is the mathematical expression of the mass conservation law in closed systems. During deformation-growth (on the right-hand side of the figure) the 'number' of material points still remains the same what allows for using the standard continuum mechanics description, however, the number of cells, molecules, and etc is changing inside the pointvolume. The latter means that the referential mass density is changing during deformation-growth: $\rho \neq$ constant, and mass is not conserved. The violation of mass conservation is inherent in open systems exchanging material with their environment.

While the qualitative analysis of the geometry and physics of tissue growth justifies the use of continuum mechanics for an open system, it is insufficient for the development of the particular equations of a macroscopic phenomenological theory. Such development requires some microscopic reasoning in order to motivate the continuum field and constitutive equations. It seems that a reasonable insight into the tissue growth mechanisms can be gained by considering a very simple toy-tissue model (see also Gordon and Jacobson, 1978). Such model is presented in Fig. 2. The regular initial tissue can be seen on the top of the figure. This is a collection of the regularly packed balls. The balls are interpreted as the tissue elementary components-cells, molecules of the extracellular matrix, and etc. The balls are arranged in a regular network for the sake of simplicity and clarity. They can be organized more chaotically - this does not affect the subsequent qualitative analysis. Let us assume now that a new material, i.e. a number of new

Fig. 1. Closed versus open systems: mass conservation versus mass flow. 
Fig. 2. Toy-tissue microscopic model: regular (top), point mass supply (bottom).

balls, is supplied pointwisely as it is shown on the bottom of Fig. 2 . This supply is considered as a result of injection: the tube with the new material is a syringe. Usually, the new material is created in real tissues in a more complicated manner following a chain of biochemical transformations. However, the finally produced new material still appears pointwisely from the existing cells. For example, the role of the material supplying tubes is played by the osteoblasts in the case of the bone tissue. Thus, the injection of the balls is a quite reason-
00000000000000 00000000000000 00000000000000 00000000000000 00000000000000 00000000000000 00000000000000 00000000000000 00000000000000

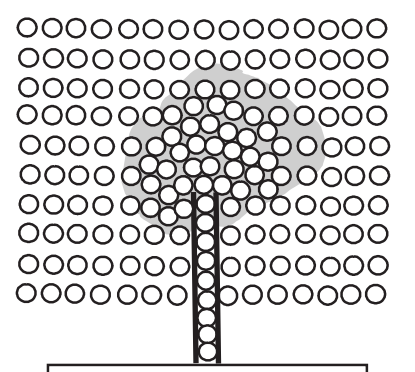

material source 00000000000000

able model of tissue growth. Such model can be constructed physically, of course. It seems that the latter is not necessary and the toy-tissue model can be easily imagined. The result of such thought-experiment is shadowed in the figure and it can be described as follows:

(a) The number of the balls in the toy-tissue increases with the supply of the new ones.

(b) The new balls are concentrated at the edge of the tube and they do not spread uniformly over the tissue.

(c) The new balls cannot be accommodated at the point of their supply - the edge of the tube: they tend to spread over the area at the vicinity of the edge and the packing of the balls gets denser around the edge of the tube.

(d) The more balls are injected the less room remains for the new ones.

(e) The new balls press the old ones.

(f) The new balls tend to expand the area occupied by the tissue when the overall ball rearrangement reaches the tissue surface.

These six qualitative features of the toy-tissue microscopic behavior under the material supply can be translated into the language of the macroscopic theory accordingly:

(A) Mass of the tissue grows.

(B) Mass growth is not uniform - the mass density changes from one point to another.

(C) There is a diffusion of mass.

(D) The diffusion is restricted by the existing tissue structure and its mass density: the denser is the tissue the less material it can accommodate.

(E) Growth is accompanied by stresses.

(F) The expansion of the tissue is volumetric - it is analogous to the thermal expansion of structural materials as steel, for example.

Three first features $(A, B$, and $C)$ prompt the form of the mass balance law in equilibrium $(\partial \rho / \partial t=0)$ :

$$
\psi_{i, i}+\xi=0
$$

where $\psi_{i}$ is the vector of mass flux and $\xi$ is the volumetric mass supply. It is convenient to manipulate equations by designating partial derivatives with respect to Cartesian coordinates $\left\{x_{1}, x_{2}, x_{3}\right\}$ with comma: $\partial() / \partial x_{i} \equiv()_{, i}$; and by summing from 1 to 3 over repeated indices: $\psi_{i, i}=\psi_{1,1}+\psi_{2,2}+\psi_{3,3}$.

Indeed, the mass change means the failure of the mass conservation law, which covers most theories of mechanics, and it means the necessity to introduce a full-scale mass balance for an open system. The fact that non-uniform mass growth is related with the diffusion of mass is very important. It means that the mass balance law should include both the volumetric mass source and the surface mass flux. The latter is missed in many theories of growth. The absence of mass diffusion in the theory leads to a nonphysical conclusion that tissue density can change only at the point of material supply, i.e. pointwisely. Mass diffusion should take place in order to accommodate a non-uniform mass supply. Boundary conditions complete the law of mass balance:

$$
\rho=\rho^{*} \text { or } \psi_{i} n_{i}=\phi^{*}
$$

where the asterisk means a given magnitude and $n_{i}$ is a unit outward vector, which is normal to the surface.

Momentum balance and corresponding boundary conditions take the traditional form in the case of a quasi-static (equilibrium) process

$$
\begin{aligned}
& \sigma_{i j, j}=0 \\
& u_{i}=u_{i}^{*} \quad \text { or } \quad \sigma_{i j} n_{j}=T_{i}^{*}
\end{aligned}
$$

where $\sigma_{i j}$ is the stress tensor; $u_{i}$ is the displacement vector; and $T_{i}$ is the surface traction.

Three last features (D, E, and F) of the toy-tissue model motivate the constitutive law. They suggest that the stress-strain relations should be analogous to thermoelasticty where the role of the temperature is played by the mass density: the increase of the mass density results in the volume expansion of the tissue:

$$
\sigma_{i j}=C_{i j m n} u_{m, n}-\left(\rho-\rho_{0}^{*}\right) \eta_{i j}
$$

where $C_{i j m n}$ is the tensor of elastic moduli enjoying minor and major symmetries; $\rho_{0}^{*}$ is the initial distribution of mass density; and $\eta_{i j}$ is a symmetric tensor of growth moduli.

On the other hand, the additional mass supply should be prevented by the tissue: the denser tissue the less is the new mass accommodation:

$$
\xi=\omega+f\left(u_{m, n}, \sigma_{i j}\right)-\left(\rho-\rho_{0}^{*}\right) \gamma
$$

where $\omega>0$ is the genetic mass supply, which is analogous to a quasi-static mechanical load (in contrast to the latter, however, $\omega$ is controlled by the tissue itself and its proper determination requires experiments); $f$ is the epigenetic mass supply ${ }^{1}$, which should depend on stress and/or strain measures (its correct expression is a key problem when tissue remodeling is considered); the last term on the right-hand side of Eq.(2.6) including coefficient of tissue resistance, $\gamma>0$, reflects the resistance of the tissue to accommodate new mass for increasing mass density

\footnotetext{
${ }^{1}$ Genetic and epigenetic mass supplies should not be confused with the genetic and epigenetic patterning. The patterning can be epigenetic even though mass supply is purely genetic.
} 
(roughly speaking, the more new material the less room for it remains).

Finally, we introduce the constitutive equation for mass flux in the simplest possible form (Fick's law):

$$
\psi_{i}=\beta\left(\rho-\rho_{0}^{*}\right)_{, i}
$$

where $\beta$ is the mass conductivity of the material.

The similarity between Eqs.(2.5) and (2.7) of growth and thermoelasticity is obvious after replacing the mass density increment by the temperature increment, mass flux by heat flux, mass conductivity by thermal conductivity, and etc. In this case Eq.(2.5) is nothing but the thermoelastic generalization of the Hooke's law, and Eq.(2.7) is just the Fourier law of heat conduction. The constitutive equation analogous to (2.6), however, is usually absent in thermoelasticity because of the lack of volumetric heat sources. The thermoelastic analogy allows for the better understanding of parameters of the growth model. For example, the vector of mass flux is analogous to the vector of heat flux. We feel the heat flow by changing temperature without directly defining what the heat is. The same is true for the mass flow. We 'feel' it by changing mass density without directly defining what it is.

Substituting the constitutive law (2.5)-(2.7) in the field equations (2.1) and (2.3) and ignoring kinetics of growth (quasi-equilibrium growth: $\partial \rho / \partial t=0)$, we get a system of coupled equations in terms of mass densities and displacements

$$
\left\{\begin{array}{l}
\beta\left(\rho-\rho_{0}^{*}\right)_{, i i}-\gamma\left(\rho-\rho_{0}^{*}\right)+f=0 \\
C_{i j m n} u_{m, n j}-\eta_{i j}\left(\rho-\rho_{0}^{*}\right)_{, j}=0
\end{array}\right.
$$

Boundary conditions (2.2) and (2.4) complete the formulation. It is important to emphasize that we tacitly assumed that deformations are small during the growth process. Such formulation is easy to understand and, besides, it is enough for our subsequent analysis. However, such formulation is not enough for the accurate setting of the incremental boundary value problem, which is

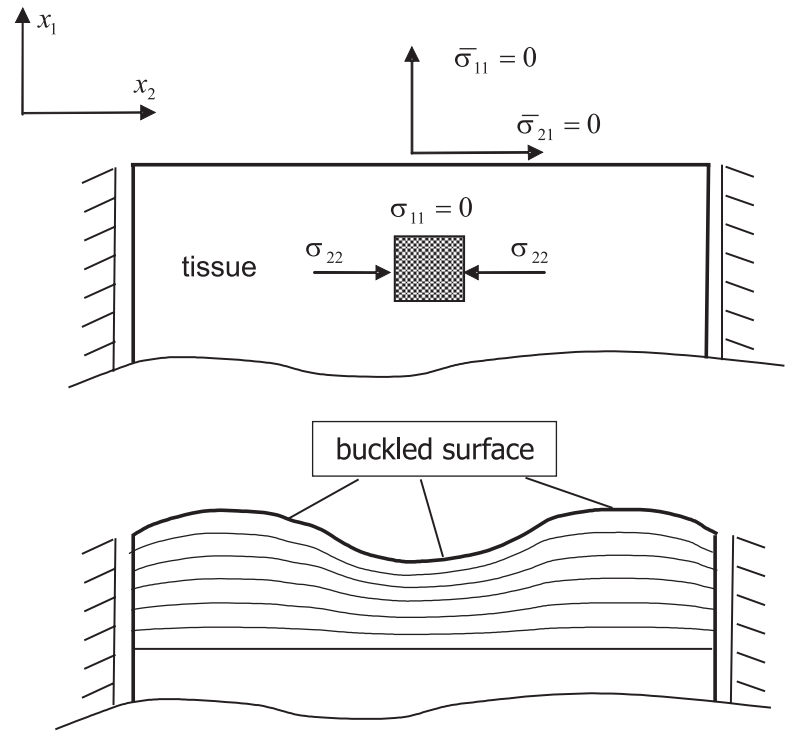

Fig. 3. An example of surface buckling in restrained tissue growth. considered below. To this end, large deformations are addressed in Appendix A.

In order to analyze surface buckling of a growing tissue we will need an incremental formulation of the considered field and constitutive equations. In general, both displacement and mass density increment should be considered. However, we will ignore the remodeling issue and drop the epigenetic factor from the equations. This allows for uncoupling the deformation/growth equations and assuming equilibrium bifurcation only, i.e. mass density is not changing because of the equilibrium bifurcation. In this case, the incremental equilibrium equations, constitutive law, and boundary conditions take the following forms accordingly (see Appendix B)

$$
\begin{gathered}
\bar{\sigma}_{i j, j}=0 \\
\bar{\sigma}_{i j}=C_{i j k n} \bar{u}_{k, n}+C_{m j k n} u_{i, m} \bar{u}_{k, n}+C_{i j m n} u_{k, m} \bar{u}_{k, n}+\sigma_{j n} \bar{u}_{i, n} \\
\bar{u}_{i}=0 \text { or } \bar{\sigma}_{i j} n_{j}=0
\end{gathered}
$$

where the bar above the letters means the corresponding increment.

\section{Example of surface buckling in volumetric growth}

We apply the general theory developed in the previous section to the problem of growth of a half plane shown in Fig. 3 (top). It is assumed that deformation of the material is restrained in the horizontal direction without defining the length of the restrained area. It is also assumed that the surface of the growing material is traction-free. In this case, we have field equations and boundary conditions in the following $2 \mathrm{D}$ form

$$
\begin{aligned}
& \psi_{1,1}+\psi_{2,2}+\xi=0 \\
& \psi_{1}\left(0, x_{2}\right)=0 \\
& \left\{\begin{array}{l}
\sigma_{11,1}+\sigma_{12,2}=0 \\
\sigma_{21,1}+\sigma_{22,2}=0
\end{array}\right. \\
& \left\{\begin{array}{l}
\sigma_{11}\left(0, x_{2}\right)=0 \\
\sigma_{21}\left(0, x_{2}\right)=0
\end{array}\right.
\end{aligned}
$$

Constitutive equations take the form

$$
\begin{aligned}
& \left\{\begin{array}{l}
\sigma_{11}=C_{1111} u_{1,1}+C_{1122} u_{2,2}-\eta_{11}\left(\rho-\rho_{0}^{*}\right) \\
\sigma_{22}=C_{2211} u_{1,1}+C_{2222} u_{2,2}-\eta_{22}\left(\rho-\rho_{0}^{*}\right) \\
\sigma_{12}=\sigma_{21}=C_{1212}\left(u_{1,2}+u_{2,1}\right)
\end{array}\right. \\
& \xi=\omega-\gamma\left(\rho-\rho_{0}^{*}\right)
\end{aligned}
$$

The following solution of the described boundary value problem can be checked by direct substitution in Eqs.(3.1)-(3.7)

$$
\left\{\begin{array}{l}
\rho-\rho_{0}^{*}=\omega / \gamma \\
u_{1,1}=\omega \eta_{11} / \gamma C_{1111} \equiv a>0 ; \quad u_{i, j}=0 \\
\sigma_{22}=-\left(\eta_{22} C_{1111} / \eta_{11}-C_{2211}\right) a=-b<0 ; \quad \sigma_{i j}=0
\end{array}\right.
$$

The physical meaning of this solution is very simple: compressive stresses appear in the horizontal direction, parallel to the free 
surface because of constrained growth of the tissue (it can expand freely in the vertical direction only). This solution describes growth of the tissue sample for the gradually increasing volumetric mass supply $\omega$. The peaceful growth lasts until the equilibrium state bifurcates producing surface patterns. In order to examine this bifurcation we have to consider the incremental boundary value problem trying to find a nontrivia/initial post-bifurcation solution of the surface buckling type.

The incremental constitutive equations take the form:

$$
\left\{\begin{array}{l}
\bar{\sigma}_{11}=C_{1111} \bar{u}_{1,1}+C_{1122} \bar{u}_{2,2}+2 C_{1111} a \bar{u}_{1,1}+C_{1122} a \bar{u}_{2,2} \\
\bar{\sigma}_{22}=C_{2211} \bar{u}_{1,1}+C_{2222} \bar{u}_{2,2}+C_{2211} a \bar{u}_{1,1}-b \bar{u}_{2,2} \\
\bar{\sigma}_{12}=C_{1212}\left(\bar{u}_{1,2}+\bar{u}_{2,1}\right)+C_{1212} a\left(\bar{u}_{1,2}+\bar{u}_{2,1}\right)+C_{1212} a \bar{u}_{1,2}-b \bar{u}_{1,2} \\
\bar{\sigma}_{21}=C_{1212}\left(\bar{u}_{1,2}+\bar{u}_{2,1}\right)+C_{1212} a \bar{u}_{1,2}
\end{array}\right.
$$

Equilibrium equations and corresponding boundary conditions can be written as

Where

$$
\begin{aligned}
& \left\{\begin{array}{l}
h_{1} \bar{u}_{1,11}+h_{2} \bar{u}_{2,21}+h_{3} \bar{u}_{1,22}=0 \\
h_{4} \bar{u}_{2,11}+h_{5} \bar{u}_{1,12}+h_{6} \bar{u}_{2,22}=0
\end{array}\right. \\
& \left\{\begin{array}{l}
\bar{\sigma}_{11}\left(x_{1}=0\right)=h_{7} \bar{u}_{1,1}+h_{8} \bar{u}_{2,2}=0 \\
\bar{\sigma}_{21}\left(x_{1}=0\right)=h_{9} \bar{u}_{2,1}+h_{10} \bar{u}_{1,2}=0 \\
\bar{u}_{1}\left(x_{1}=-\infty\right)=0 \\
\bar{u}_{2}\left(x_{1}=-\infty\right)=0
\end{array}\right.
\end{aligned}
$$

$$
\left\{\begin{array}{l}
h_{1}=\left(C_{1111}+2 C_{1111} a\right) / C_{1111} \\
h_{2}=\left(C_{1122}+C_{1212}+C_{1122} a+C_{1212} a\right) / C_{1111} \\
h_{3}=\left(C_{1212}+2 C_{1212} a-b\right) / C_{1111} \\
h_{4}=C_{1212} / C_{1111} \\
h_{5}=\left(C_{1212}+C_{1212} a+C_{2211}+C_{2211} a\right) / C_{1111} \\
h_{6}=\left(C_{2222}-b\right) / C_{1111} \\
h_{7}=\left(C_{1111}+2 C_{1111} a\right) / C_{1111} \\
h_{8}=\left(C_{1122}+C_{1122} a\right) / C_{1111} \\
h_{9}=C_{1212} / C_{1111} \\
h_{10}=\left(C_{1212}+C_{1212} a\right) / C_{1111}
\end{array}\right.
$$

We present solution in the form

$$
\left\{\begin{array}{l}
\bar{u}_{1}=y_{1}\left(x_{1}\right) \cos \theta x_{2} \\
\bar{u}_{2}=y_{2}\left(x_{1}\right) \sin \theta x_{2}
\end{array}\right.
$$

which allows for the wavy pattern formation in the horizontal direction.

Substituting (3.13) in (3.10)-(3.11) we have the following equilibrium equations and boundary conditions accordingly

$$
\left\{\begin{array}{l}
h_{1} \frac{\partial^{2} y_{1}}{\partial x_{1}^{2}}+\theta h_{2} \frac{\partial y_{2}}{\partial x_{1}}-\theta^{2} h_{3} y_{1}=0 \\
h_{4} \frac{\partial^{2} y_{2}}{\partial x_{1}^{2}}-\theta h_{5} \frac{\partial y_{1}}{\partial x_{1}}-\theta^{2} h_{6} y_{2}=0
\end{array}\right.
$$

$$
\left\{\begin{array}{l}
\bar{\sigma}_{11}\left(x_{1}=0\right)=h_{7} \frac{\partial y_{1}}{\partial x_{1}}+\theta h_{8} y_{2}=0 \\
\bar{\sigma}_{21}\left(x_{1}=0\right)=h_{9} \frac{\partial y_{2}}{\partial x_{1}}-\theta h_{10} y_{1}=0 \\
\bar{u}_{1}\left(x_{1}=-\infty\right)=0 \\
\bar{u}_{2}\left(x_{1}=-\infty\right)=0
\end{array}\right.
$$

Let us consider solution of (3.14) and (3.15) in the form

$$
\left\{\begin{array}{l}
y_{1}=A \exp \left(r \theta x_{1}\right) \\
y_{2}=B \exp \left(r \theta x_{1}\right)
\end{array}\right.
$$

which means decay of the deformation and stresses away from the surface in the vertical direction.

Substituting (3.16) in (3.14) we get

$$
\left\{\begin{array}{l}
\left(h_{1} r^{2}-h_{3}\right) A+h_{2} r B=0 \\
-h_{5} r A+\left(h_{4} r^{2}-h_{6}\right) B=0
\end{array}\right.
$$

A nontrivial solution of this system of equations is obtained when

$$
h_{1} h_{4} r^{4}+\left(h_{5} h_{2}-h_{3} h_{4}-h_{1} h_{6}\right) r^{2}+h_{6} h_{3}=0
$$

This equation has four roots $r_{1},-r_{1}, r_{2},-r_{2}$. If they are real then we can choose two positive roots, say $r_{1}, r_{2}>0$, and entirely omit negative roots what satisfies boundary conditions on infinity $\left(3.15_{3,4}\right)$. Substituting these roots in Eq.(3.17) we can find coefficient $A$ as a function of coefficient $B$.

$$
\left\{\begin{array}{l}
A_{1}=H_{1} B_{1}, \quad H_{1}=\left(h_{4} r_{1}^{2}-h_{6}\right) / h_{5} r_{1} \\
A_{2}=H_{2} B_{2}, \quad H_{2}=\left(h_{4} r_{2}^{2}-h_{6}\right) / h_{5} r_{2}
\end{array}\right.
$$

Thus the solution of Eq.(3.14) with boundary conditions $(3.153,4)$ can be written as

$$
\left\{\begin{array}{l}
y_{1}=H_{1} B_{1} \exp \left(r_{1} \theta x_{1}\right)+H_{2} B_{2} \exp \left(r_{2} \theta x_{1}\right) \\
y_{2}=B_{1} \exp \left(r_{1} \theta x_{1}\right)+B_{2} \exp \left(r_{2} \theta x_{1}\right)
\end{array}\right.
$$

It remains to satisfy boundary conditions on the free surface. Substituting $(3.20)$ in $\left(3.15_{1,2}\right)$ we have

$$
\left\{\begin{array}{l}
\left(h_{7} H_{1} r_{1}+h_{8}\right) B_{1}+\left(h_{7} H_{2} r_{2}+h_{8}\right) B_{2}=0 \\
\left(h_{9} r_{1}-h_{10} H_{1}\right) B_{1}+\left(h_{9} r_{2}-h_{10} H_{2}\right) B_{2}=0
\end{array}\right.
$$

This system of equations possesses a nontrivial solution when

$g(a)=\left(h_{7} H_{1} r_{1}+h_{8}\right)\left(h_{9} r_{2}-h_{10} H_{2}\right)-\left(h_{7} H_{2} r_{2}+h_{8}\right)\left(h_{9} r_{1}-h_{10} H_{1}\right)=0$

Consider a material with the following elasticity and growth parameters:

$C_{1111}=1 ; \quad C_{2222}=100 ; \quad C_{1122}=C_{2211}=0.5 ; \quad C_{1212}=1 ; \quad \eta_{11}=1 ; \quad \eta_{22}=1$ 
It should not be missed that the dimension of this parameters is of no interest because only their relative magnitudes matter. In this case the smallest positive root of Eq.(3.22) is

$$
a \approx 20
$$

which corresponds to the positive solution of Eq.(3.18) and, consequently, provides the desirable surface buckling solution for the initial post-bifurcation equilibrium.

It is worth emphasizing that the 'surface' solution of the incremental problem does not necessarily exist. For example, in the case of material isotropy, where ${ }^{2} \quad C_{i j m n}=\lambda \delta_{i j} \delta_{m n}+2 \mu \delta_{i m} \delta_{j n}$ the 'surface' solution does not exist for various reasonably chosen values of Lame parameters $\lambda$ and $\mu$.

\section{Discussion and conclusions}

A novel mechanism of tissue morphogenesis has been proposed. According to it, surface patterns can emerge during growth of anisotropic tissues because of surface buckling. This scenario has been examined based on a simple phenomenological theory of tissue growth. It is shown, particularly, that constrained growth can lead to tissue compression, which, in its turn, may result in surface buckling of the tissue. The latter means the appearance of wavy patterns on the surface. These patterns decay away from the surface. It is interesting that the critical magnitude of the mass supply parameter, which corresponds to the surface buckling, is independent of the pattern wavelength and, generally, various patterns can be generated in growth. The results of the performed theoretical analysis show that the surface buckling scenario is realistic if the growing tissue matches two following conditions. First, compression should appear during tissue growth. Second, the tissue should exhibit strong anisotropy. The former condition does not necessarily mean geometric constraints: inhomogeneous growth or material inhomogeneity and anisotropy can lead to the appearance of compressive stresses (see below). The latter condition is typical of some tissues with fiber reinforcement in planes parallel to the surface. In this case, the tissue material is much softer in the out-of-plane direction than in plane.

It is worth noting again that we considered an example where compression appeared because of the tissue geometric constraint, what physically means interaction among various tissues. However, compressive stresses can appear in a different way without the tissue interactions. Let us consider a growth scenario where material is supplied at the free surface (surface growth). In this case, we set $\omega=0$ in the governing equations and impose new boundary conditions on the mass density at the free surface $\rho\left(x_{1}=0\right)=\rho_{0}^{*}+\rho^{*}$ where a parameter of the surface mass supply is $\rho^{*}$. In this case, the growing half plane, considered above, can be described by the following solution of the corresponding boundary value problem:

$$
\left\{\begin{array}{l}
\rho-\rho_{0}^{*}=\rho^{*} \exp \left(\tau x_{1}\right) ; \quad \tau=\sqrt{\gamma / \beta} \\
u_{1}=\left(\rho^{*} \eta_{11} / \tau C_{1111}\right) \exp \left(\tau x_{1}\right)>0 ; \quad u_{i, j}=0 \\
\sigma_{22}=-\left(\eta_{22}-\eta_{11} C_{2211} / C_{1111}\right) \exp \left(\tau x_{1}\right)<0 ; \quad \sigma_{i j}=0
\end{array}\right.
$$

${ }^{2}$ We use the Kronecker delta defined as $\begin{cases}\delta_{i j}=1, & i=j \\ \delta_{i j}=0, & i \neq j\end{cases}$
This solution is of the boundary layer type, that is nonzero density, stress, and displacement fields are concentrated near the surface and they decay away from the surface. The properly defined elastic parameters may lead to the appearance of the compressive stresses parallel to the free surface $\left(x_{1}=0\right)$. Thus, inhomogeneous growth is a constrained growth: material of the boundary layer tends to expand while the bulk material prevents from this expansion. It would be interesting to analyze the bifurcation and possible buckling of the surface in this case. Unfortunately, this is not simple because the coefficients of the incremental boundary value problem get inhomogeneous and a direct analytical solution is hardly available. Numerical analysis is desirable.

One of the remarkable features of the obtained analytical solution is the fact that the bifurcation of the equilibrium can create unlimited and unpredictable variety of tissue patterns because the critical parameter of mass supply does not depend on the pattern wavelength. This fact may be a consequence of some simplifying assumptions made in the analysis and, probably, a numerical solution of a more realistic example would give a finite bifurcation multiplicity. However, the interpretation of the possible variety of tissue patterns can have a deeper meaning. It is likely that we are just unable to decide about the specific patterning based on the epigenetic scenario only. It can occur that nature decides about specific patterns through a genetic mechanism, which was not included in our consideration. This circle of questions remains highly speculative, of course.

Finally, we have to emphasize that the considered surface buckling mechanism of the tissue patterning is a purely theoretical prediction yet. It could be a challenging experimental problem to create patterns by restraining tissue growth and forcing the surface to buckle.

\section{References}

BARD, J. (1990). Morphogenesis. Cambridge University Press, Cambridge.

BIOT, MA. (1965). Mechanics of Incremental Deformations. Wiley, New York.

COWIN, SC. (2001). Bone Mechanics Handbook. 2nd edn. CRC Press, Boca Raton.

COWIN, SC. (2004). Tissue growth and remodeling. Annual Review of Biomedical Engineering. 6, 77-107.

COWIN, SC., and HEGEDUS, DH. (1976). Bone remodeling I: Theory of adaptive elasticity. Journal of Elasticity. 6, 313-326.

GORDON, R., and JACOBSON, AG. (1978). The shaping of tissues in embryos. Scientific American. 238 (6), 106-113.

GREEN, PB. (1999). Expression of pattern in plants: combining molecular and calculus-based biophysical paradigms. American Journal of Botany. 86, 1059-76.

MURRAY, JD. (2003). Mathematical Biology. 3rd edn. Springer, Berlin.

NEWMAN, SA., and Muller, GB. (2000). Epigenetic mechanisms of character origination. Journal of Experimental Zoology. 288, 304-17.

OGDEN, RW. (1984). Non-Linear Elastic Deformations. Ellis Horwood, Chichister.

STEELE, CR. (2000). Shell stability related to pattern formation in plants. ASME Journal of Applied Mechanics. 67, 237-247.

TRUESDELL, C., and NOLL, W. (1965). The Non-Linear Field Theories of Mechanics. In: Flugge S (Ed.): Encyclopedia of Physics, Vol. III/3. Springer, Berlin.

TURING, AM. (1952). The chemical basis of morphogenesis. Philosophical Transactions of the Royal Society of London B. 23, 37-72.

VOLOKH, KY. (2004a). Mathematical framework for modeling tissue growth. Biorheology. 41, 263-269. 
VOLOKH, KY. (2004b). A simple phenomenological theory of tissue growth. Mechanics and Chemistry of Biosystems. 1, 147-160.
VOLOKH, KY., and LEV, Y. (2005). Growth, anisotropy, and residual stresses in arteries. Mechanics and Chemistry of Biosystems. 2, 27-40.

\section{Appendix A}

According to the general setting of continuum mechanics (Truesdell and Noll, 1965; Ogden, 1984) a material point occupying position $X_{1}$ in the reference configuration moves to position $X_{i}$ in the current configuration. Equilibrium equations and the corresponding boundary conditions take the following form

$$
\begin{aligned}
& P_{i l, l}=0 \\
& x_{i}=x_{i}^{*} \quad \text { or } \quad P_{i l} n_{l}=T_{i}^{*}
\end{aligned}
$$

where $P_{i l}$ are the components of the 1st Piola-Kirchhoff stress tensor, which can be interpreted as the $i$ th component of force per unit reference area on an element of surface in the current configuration whose normal was in the / th direction in the reference configuration.

Equations of the steady-state mass balance and the corresponding boundary conditions can be written as follows

$$
\begin{aligned}
& \psi_{l, l}+\xi=0 \\
& \rho=\rho^{*} \text { or } \quad \psi_{l} n_{l}=\phi^{*}
\end{aligned}
$$

Constitutive equations can be written in a general form

$$
\begin{aligned}
& P_{i l}=P_{i l}\left(x_{i, J}, \rho, \rho_{, K}, X_{M}\right) \\
& \psi_{l}=\psi_{l}\left(x_{i, J}, \rho, \rho_{, K}, X_{M}\right) \\
& \xi=\xi\left(x_{i, J}, \rho, \rho_{, K}, X_{M}\right)
\end{aligned}
$$

The described deformation/growth boundary value problem follows the so-called Lagrangean formulation where reference coordinates $X_{1}$ are independent variables. Such formulation is convenient for solids while fluids are generally described following the Euler formulation with $x_{i}$ as independent variables. Of course, both formulations are physically equivalent being mathematically different.

If the deformation is small then it is necessary to substitute $x_{i, l}=\delta_{i l}+u_{i, l}$ in the above equations and to ignore the small terms $\left(u_{i, l}\right)$ properly. In this case, the difference between the Lagrangean and Eulerian formulations disappears.

\section{Appendix B}

Let us consider the incremental equilibrium (see also the pioneering work by Biot, 1963) of the body using the general setting of Appendix A. In this case

and

$$
\bar{P}_{i l, l}=0
$$

$$
\bar{x}_{i}=\bar{u}_{i}=0 \text { or } \bar{P}_{i l} n_{l}=0
$$

where the increments are barred. Incremental constitutive law takes the form

$$
\bar{P}_{i l}=\frac{\partial P_{i l}}{\partial x_{m, J}} \bar{x}_{m, J}=B_{i \mid \mathrm{m} J} \bar{u}_{m, J}
$$

$$
B_{i \mid \mathrm{m} J}=\frac{\partial P_{i l}}{\partial x_{m, J}}=\frac{\partial\left(S_{K I} x_{i, K}\right)}{\partial x_{m, J}}=\frac{\partial S_{K l}}{\partial E_{L M}} \frac{\partial E_{L M}}{\partial x_{m, J}} x_{i, K}+S_{K l} \frac{\partial x_{i, K}}{\partial x_{m, J}}=C_{L I K J} x_{i, L} x_{m, K}+\delta_{i m} S_{I J}
$$

where use having been made of the result

$$
\frac{\partial E_{L M}}{\partial x_{m, J}}=\frac{\partial\left(x_{i, L} x_{i, M}-\delta_{L M}\right) / 2}{\partial x_{m, J}}=\frac{1}{2}\left(\delta_{L J} x_{m, M}+\delta_{M J} x_{m, L}\right)
$$

The symmetric 2nd Piola-Kirchhoff stress tensor $S_{K l}$ introduced above does not enjoy clear physical interpretation (except for the practically important case of small strains and large rotations of slender bodies). The tensor of elastic moduli $C_{I J K L}=\partial S_{I J} / \partial E_{K L}$ relating the 2 nd Piola-Kirchhoff stress tensor $S_{l J}$ with the Green strain tensor $E_{K L}$ generally depends on deformation. Now the stress increments take the form

$$
\bar{P}_{i l}=C_{L I K J}\left(\delta_{i L}+u_{i, L}\right)\left(\delta_{m K}+u_{m, K}\right) \bar{u}_{m, J}+S_{I J} \bar{u}_{i, J}
$$

In the case of small deformations the terms quadratic in displacement gradients can be ignored

$$
\bar{P}_{i l} \approx C_{L I K J}\left(\delta_{i L} \delta_{m K}+\delta_{m K} u_{i, L}+\delta_{i L} u_{m, K}\right) \bar{u}_{m, J}+S_{I J} \bar{u}_{i, J}
$$

as well as the difference between various stress tensors and upper and lower case indices. 